\title{
Estándares para la Investigación sobre Pruebas Proyectivas y Abuso Sexual Infantil
}

\author{
Standards for Research on Projective Tests and Child Sexual Abuse
}

\author{
Carolina Navarro ${ }^{1}$, Iris Gallardo ${ }^{2}$ y Ruth Weinstein ${ }^{3}$
}

\begin{abstract}
Resumen
La validez de las pruebas proyectivas en la evaluación de víctimas de abuso sexual infantil no ha sido suficientemente establecida, siendo un ámbito controversial. A partir de una revisión crítica de la literatura disponible, el presente artículo argumenta la existencia de deficiencias en la investigación en este campo. De este análisis se deriva una propuesta metodológica para el diseño de estudios empíricos cuantitativos que busquen establecer la validez de los instrumentos proyectivospara su uso en esta población. Esta propuesta, que consideralos principios de cientificidad, la naturaleza de las técnicas proyectivas como instrumentos psicodiagnósticos y la complejidad del fenómeno del abuso sexual, provee un modelo metodológico operacional para el desarrollo de una línea de investigación comparable y de calidad en este campo. Finalmente, se discuten las implicancias y alcances de la propuesta formulada.
\end{abstract}

Palabras clave: pruebas gráficas, pruebas narrativas, psicodiagnóstico, abuso sexual, niños víctimas

\begin{abstract}
The validity of projective tests for evaluating child sexual abuse victims has not been established and remains controversial. From a critical review of available literature, the present article argues the existence of deficiencies in research in this field. From this analysis derived a methodological proposal for the design of quantitative empirical studies that seek to establish the validity of projective tests for their use with this population. This proposal, which considers the principles of scientificity, the nature of projective techniques as psychodiagnostic instruments and the complexity of the phenomenon of sexual abuse, provides an operational methodological model for the development of a comparable line of research in this field. Finally, the implications and scope of the proposal formulated are discussed.
\end{abstract}

Keywords: drawing tests, narrative tests, psychodiagnosis, sexual abuse, child victims

La preparación de este artículo fue financiada por el Programa de Apoyo a la Productividad Académica, PROA VID 2015; Universidad de Chile.

\footnotetext{
${ }^{1}$ PhD. Profesora Asistente, Departamento de Psicología, Universidad de Chile. Av. Ignacio Carrera Pinto $10454^{\circ}$ piso, Ñuñoa, Santiago, Chile. Tel.: (56-2) 29787878. Correo: cnavarro@u.uchile.cl

${ }^{2}$ Magister en Psicología. Profesora Asistente, Departamento de Psicología, Universidad de Chile. Av. Ignacio Carrera Pinto $10454^{\circ}$ piso, Nuñoa, Santiago, Chile. Tel.: (56-2) 29787878. Correo: i.gallardorayo@ gmail.com

${ }^{3}$ Magister en Psicología, Profesora Asistente, Departamento de Psicología, Universidad de Chile. Av. Ignacio Carrera Pinto $10454^{\circ}$ piso, Nuñoa, Santiago, Chile. Tel.: (56-2) 29787878. Correo: ruth.weinstein.a@gmail.com
} 


\section{Introducción}

El abuso sexual infantil (ASI) es un problema social de amplias implicancias, pero que mayormente preocupa debido a su impacto en el bienestar de las víctimas. La investigación en este campo ha demostrado que la violencia sexual contra niños, niñas y adolescentes (NNA) produce, en gran parte de quienes lo sufren, reacciones emocionales y físicas adversasen el corto plazo (Finkelhor \& Browne, 1985; CollinVézina, Daigneault, \& Hébert, 2013), así como consecuencias psicológicas de largo plazo que pueden evidenciarse incluso en la vida adulta (Hillberg, Hamilton-Giachritsis, \& Dixon, 2011). Esto hace del ASI un problema relevante en el ámbito de la salud mental que demanda intervenciones orientadas a favorecer la recuperación de la víctima.

Por otro lado, el ASI constituye una grave vulneración de derechos cuya denuncia da lugar a intervenciones judiciales de distinta índole. Tanto para la justicia penal como para la proteccional, la ausencia de pruebas materiales y de testigos que caracteriza este tipo de delitos representa una importante dificultad a la hora de probar la existencia del abuso. Como resultado, el sistema de justicia ha demandado de manera creciente por intervenciones psicológicas forenses que ofrezcan evidenciade que el NNA ha sufrido abuso.

En este contexto, la evaluación psicológica orientada a establecer el impacto del abuso en la víctima resulta crucial tanto en la planificación de intervenciones reparatorias, como enla aplicación de justicia. Sin embargo, la evaluación psicológica en esta población presenta importantes desafíos diagnósticos. Por un lado, la inespecificidad de los síntomas que presentan las víctimas y, por otro, la dificultad que muestran muchos NNA para relatar su experiencia abusiva, hacen que el uso de estrategias diagnósticas basadas en los niveles sintomatológico y/o discursivo presente una utilidad limitada. El uso de métodos de evaluación que no dependan de la capacidad del NNA para relatar la experiencia abusiva o de la posibilidad de identificar determinados síntomas, resulta una necesidad. Es aquí donde el uso de instrumentos psicológicos juega un rol relevante como herramienta de evaluación.
Es preciso señalar que no existen instrumentos conocidos específicos para la detección del abuso ni para la evaluación del impacto psicológico en NNA $^{1}$. Frente a esta carencia, los profesionales de la Psicología han recurrido al uso de diferentes instrumentos, dentro de los que destacan las pruebas proyectivas (PP). Entre las más utilizadas se cuentan las PP gráficas Dibujo de la Figura Humana, el Dibujo de la Familia, el Test ÁrbolCasa-Persona (HTP) y el Dibujo de la Persona Bajo la Lluvia; mientras que dentro de las PP narrativas de mayor uso se incluyen el Test de Apercepción Infantil (CAT-A y CAT-H), así como el Test de Relaciones Objetales (TRO), entre otras. Estas técnicas presentan importantes ventajas ya que permiten un contacto no amenazante y cercano al modo lúdico de funcionamiento de niños pequeños y escolares, además de favorecer una disminución de los mecanismos de defensa en NNA reacios a relatar el abuso. Estas características las han llevado a ser una herramienta diagnóstica complementaria de amplio uso por parte de psicólogos clínicos y forenses en laevaluación de víctimas de ASI.

Este interés se ha visto también reflejado en la investigación científica, tal como lo demuestra el importante volumen de estudios desarrollados desde los años $80^{\prime}$ en diferentes países. Este desarrollo ha dado lugar a un cúmulo de información publicada que delimita un campo con una historia y evolución. Esta historia se inicia con la publicación de los primeros informes de casos de investigación no controlada que planteaban que la interpretación clínica era válida para identificar signos de abuso (Allen \& Tussey, 2012), lo que dio lugar al desarrollo de guías para la detección de casos de ASI a partir de los dibujos de NNA (por ej. Hackbarth, Murphy \& McQuary, 1991citado en Allen \& Tussey, 2012). Desde entonces, se ha avanzado en rigor científico a partir del desarrollo de numerosos estudios empíricos con diseños controlados. Más recientemente, se han publicado estudios

\footnotetext{
${ }^{1}$ Como excepción, el "Inventario de Frases Abuso y Maltrato Infantil" y la "Hora de juego diagnostica", de las autoras argentinas de Agosta, Barilari y Colombo (2005) y Colombo y Beigbeder (2003) respectivamente, han sido desarrollados para la evaluación de víctimas de maltrato infantil, incluyendo abuso sexual, aunque no de manera específica.
} 
secundarios consistentes en revisiones sistemáticas (RS) y meta-análisis (MA), los que han buscado establecer el estado del conocimiento en este campo (Allen \& Tussey, 2012; Garb, Wood, \& Nezworski, 2000; Trowbridge, 1995; West, 1998).

Un análisis de la investigación disponible revela que los estudios sobre $\mathrm{PP}$ y $\mathrm{ASI}$ han usado mayoritariamente como paradigma de investigación el modelo de la identificación de indicadores proyectivos de ASI. Estos indicadores corresponderían a características específicas de los dibujos, narrativas y respuestas al test de Rorschach, que se dan con mayor frecuencia en este grupo que en otros. Ejemplos de indicadores gráficos de ASI descritos en la literatura son el dibujo de partes del cuerpo desorganizadas, genitales, omisión o sobre énfasis de partes del cuerpo, como ojos, brazos, manos, piernas, pies, boca o cuello, entre otros (Allen \& Tussey, 2012). La noción de indicadores proyectivos de ASI surge en su inicio a partir de nomenclaturas clásicas, como los indicadores emocionales de Koppitz (1980) en el caso de las pruebas gráficas y de la sistematización de la práctica clínica, siendo luego sujeto de estudios exploratorios en esta población. Este paradigma de investigación se basa en dos supuestos: (1) que existen indicadores proyectivos propios de NNA que han sufrido abuso sexual y/o maltrato, y (2) que éstos permiten diferenciar a estas víctimas de otras poblaciones de NNA (Trowbridge, 1995; West, 1998).

Tanto los estudios empíricos primarios como las revisiones se han centrado en establecer o descartar la validez de los llamados indicadores específicos de ASI. Algunos de estos trabajos han establecido la validez de estos indicadores (West, 1998), mientras que otros concluyen en un sentido contrario señalando la falta de respaldo empírico que los avale (Trowbridge, 1995; Allen \& Tussey, 2012). La discordancia de estos resultados da cuenta de un campo de investigación todavía controversial.

La falta de un cuerpo de conocimiento sólido y homogéneo en cuanto al uso de las PP para evaluar víctimas de ASI, ha introducido un importante grado de confusión en la comunidad científica y entre los profesionales de la Psicología Clínica y Forense. Esto ha dado lugar, además, a un cuestionamiento sobre el respaldo empírico que tiene esta extendida práctica profesional (Scott, Manzanero, Muñoz, \& Köhnken, 2014). Lo anterior obliga a una reflexión crítica respecto del estudio actual en la materia, orientada a identificar las debilidades que requieren ser subsanadas por la investigación futura.

El presente artículo responde a la pregunta sobre cómo producir evidencia científica de calidad respecto a la validez de las PP en la evaluación de víctimas de ASI. Sus objetivos son argumentar la existencia de deficiencias en la investigación en este campo y presentar una propuesta metodológica para el diseño de estudios empíricos que busquen establecer la validez de las PP en esta población. Para esto, en el apartado II analizaremos tres deficiencias que presenta la investigación en la materia, conceptualizadas como brechas entre un determinado conocimiento establecido y el diseño utilizado por los estudios conocidos. En el apartado III, presentaremos una propuesta metodológica desarrollada por el Equipo de Investigación sobre Psicodiagnóstico y Violencia Sexual de la Universidad de Chile. Esta propuesta busca subsanar las deficiencias observadas en la investigación actual sobre PP y ASI. Finalmente, se discuten las implicancias y alcances de la propuesta formulada, la que esperamos contribuirá al desarrollo futuro de una línea de investigación sistemática basada en estudios de diseños metodológicos comparables.

\section{Brechas en la investigación empírica sobre el uso de las PP en la evaluación de víctimas de ASI}

La argumentación aquí presentada surge de una revisión crítica de los estudios empíricos y secundarios sobre PP y ASI publicados. Para esta revisión buscamos identificar potenciales artículos sobre RS y MA en este campo publicados entre 1995 y 2015. Usamos estrategias de búsqueda (Furlan, Pennick, Bombardier, \& van Tulder, 2009) para las bases de dato MEDLINE vía PubMed, PsycINFO y Web of Science con los téminos "child sexual abuse" y "projective tests o drawing tests o thematic tests" combinadas con "systematicre view o meta-analysis" en español e inglés. Los artículos fueron seleccionados según título, resumen y palabras clave. Adicionalmente realizamos una revisión de las referencias 
bibliográficas de los artículos identificados, así como de artículos empíricos posteriores a 2010. Seis artículos fueron identificados, dos de los cuales fueron excluidos por ser revisiones no sistemáticas ni exhaustivas. Finalmente, 4 artículos que satisfacían los criterios de búsqueda definidos fueron seleccionados, 2 RS y 2 MA. El primero correspondía a una RS de estudios empíricos que examinaran la presencia de indicadores gráficos de abuso sexual en dibujos de niños (Trowbridge, 1995); otro, fue el primer MA que buscó evaluar la efectividad de las PP para identificar ASI y maltrato (West, 1998); un tercer artículo correspondía a un nuevo MA de los datos presentados en el primer MA (Garb, Wood, \& Nezworski, 2000b), mientras que el trabajo más reciente correspondió a una RS de estudios controlados sobre pruebas gráficas para detectar abuso sexual o físico (Allen \& Tussey, 2012). Considerando solo los estudios primarios relativos a ASI, en conjunto estos 4 trabajos analizan un total de 25 estudios publicados entre 1984 y 2007 que utilizaron 9 PP y otras técnicas proyectivas (distintos tipos de dibujos) para evaluar víctimas de ASI. La Tabla A (ver Apéndice A) presenta un resumen de estos estudios primarios. La revisión crítica de la literatura identificada condujo a la construcción de un argumento cuya idea central es que el grado de ajuste que presentan los estudios empíricos sobre el PP y ASI al conocimiento científico afianzado, resulta un indicador de su calidad. Tal como ha sido señalado (por ej. Allen \& Tussey, 2012), esta investigación ha adolecido de importantes deficiencias, las que, aquí argumentamos, son resultado de una insuficiente consideración por parte de los estudios hasta ahora conducidos de conocimientos provenientes del campo de la Psicología, el Psicodiagnóstico y la Metodología de la investigación científica. Esta falta de consideración representa una distancia entre el modo en que se ha conducido la investigación sobre PP y ASI y los saberes ya afianzados en estos tres campos dando lugar a tres brechas. La identificación de estas brechas resulta útil para la formulación de nuevas propuestas que subsanen las falencias identificadas a través de diseños de investigación que sean coherentes con el conocimiento existente. En este apartado analizamos cada una de estas tres brechas.

\section{Primera brecha}

El fenómeno del ASI y sus consecuencias: distancia entre el conocimiento psicológico y la investigación

El ASI ha sido objeto de un amplio estudio que sistemáticamente ha analizado las características del fenómeno y sus consecuencias. Esto ha dado lugar al desarrollo de un sólido marco teórico empírico sobre la fenomenología de la violencia sexual contra NNA y su impacto en la salud mental de las víctimas.

El uso de las PP en la población de víctimas de ASI busca contribuir a la evaluación de las consecuencias psicológicas del abuso en las víctimas, las que han sido conceptualizadas en la literatura como daño asociado al abuso. En este trabajosos tenemos que el daño que produce el ASI es el resultado de la interacción de variables individuales y contextuales que se conjugan produciendo un cierto efecto en la víctima. Esta noción compleja de daño se deriva de la teoría del trauma y de su relación con la fenomenología del ASI.

\section{La noción de daño y la fenomenología del ASI}

La literatura sobre ASI coincide en señalar una falta de regularidad en las manifestaciones psicopatológicas que se observan en las víctimas con posterioridad al abuso (Collin-Vézina et al.,2013). Por el contrario, la ocurrencia del abuso se ha asociado a cuadros que incluyen desde síntomas ansiosos y/o conductuales hasta alteraciones permanentes de la personalidad, así como a aumento de riesgo de múltiples desordenes psiquiátricos (Chen et al., 2010; Ramírez \& y Fernández, 2011; Foster \& Carson, 2013), mientras quehasta un tercio de los NNA que han sufrido abusos sexuales no presentaría sintomatología evidente en el corto plazo, siendo estos casos considerados como resilientes (AdlerNevo \& Manassis 2005; Marriott, HamiltonGiachritsis, \& Harrop, 2014). Junto con esto, no se han identificado conductas o trastornos específicos que permitan diferenciar las victimas de ASI de otro tipo de víctimas (Kendall-Tackett, Williams, \& Finkelhor, 1993).

Lo anterior lleva a caracterizar el daño psicológico que produce el ASI como un proceso psicopatológico complejo que requiere la identificación de las variables que median su 
manifestación. Al respecto, los especialistas coinciden en que el daño que sufren las víctimas de ASI no se explica únicamente por la experiencia de abuso. Han sido identificadas una serie de variables que median la experiencia primaria de abuso provocando un daño específico en la víctima según su edad, tipo de abuso, vínculo con el agresor, reacción de la madre frente a la develación, entre otros (Ramírez \& Fernández, 2011; Foster \& Carson, 2013). De este modo, cuestiones relacionadas con la forma en que se presenta la situación abusiva, es decir, aspectos fenomenológicos, así como variables de la víctima se entrecruzan para dar lugar a efectos diferenciales.

Respecto de las variables que caracterizan el ASI, éste ha sido reconocido como un fenómeno no homogéneo (Navarro, 2011; Sawyer \& Hansen, 2014). Una de las primeras distinciones fenomenológicas que la literatura especializada reconoce respecto del ASI, es la clasificación de distintos tipos de abuso según el vínculo entre víctima y agresor. Se distingue así el abuso por parte de agresor desconocidoo conocido, subdividiéndose este último en abuso extrafamiliare intrafamiliar. Dentro de éste último se incluyen los abusos incestuosos, aquellos en que el agresor ocupa un rol parental para la víctima (Navarro, 2011). Esta distinción básica se expresa en diferencias tanto en el tipo de abuso, las estrategias utilizadas por el agresor, las características de la víctima y su agresor, así como las consecuencias del abuso para la víctima.

De acuerdo esto, el daño que puede experimentar una adolescente que ha sufrido un episodio único de violación por parte de un desconocido mediante uso de la fuerza física, se diferenciará sustancialmente de aquel que podría sufrir una niña de ocho años que ha vivido un abuso sistemático por parte de su padre desde los tres años en un contexto de manipulación afectiva. Esto, que resulta comprensible desde un punto de vista teórico, ha sido avalado por diversos estudios que han demostrado la relación entre distintas variables fenomenológicas del ASI y la magnitud del daño psicológico en las víctimas. Por ejemplo, Huerta, Maric y Navarro (2002) identificaron que, en casos de abusos incestuosos, la combinación de abuso crónico con tolerancia de la madre frente al abuso, daba lugar a daños psicológicos de mayor gravedad en las víctimas. Coincidentemente, Agosta, Barilari y Colombo (2005) señalan que todo niño sometido a un abuso intrafamiliar crónico sufre un daño psíquico que dificulta su adaptación y su normal desarrollo evolutivo.

A la luz del conocimiento disponible sobre ASI y daño en las victimas, un análisis de los estudios primarios contenidos en las RS sobre PP y ASI, revela dos problemas (ver Apéndice A). Primero, la investigación en este campo ha buscado identificar alteraciones regulares en los protocolos de NNA víctimas de abuso sexual, típicamente operacionalizadas como indicadores proyectivos de ASI. Esta aproximación contrasta con la comprensión del daño como proceso psicopatológico complejo, resultando inadecuada a la naturaleza del objeto de estudio. En segundo lugar, estos estudios primarios escasamente han incorporado como variables en sus diseños distinciones fenomenológicas que han sido identificadas como relevantes (vínculo con el agresor, tipo de abuso, frecuencia, entre otras). Por el contrario, 22 de los 25 estudios empíricos analizados por las RS, es decir un $88 \%$, no reportan distinciones en este ámbito. Los otros tres corresponden a estudios que solo consignaron el vínculo con el agresor en la descripción de sus muestras; de estos, solo uno incluyó esta distinción en el análisis de los resultados. Hackbarth et al. (1991, citado en Allen \& Tussey, 2012) analizaron los Dibujos de la Familia Kinética de un grupo de víctimas en que el abusador era un miembro de la familia, concluyendo que esta variable podía influir en los resultados. Curiosamente, esta composición homogénea de la muestra fue considerada por Allen y Tussey (2012) como una limitación del estudio. De este modo, es posible asumir que de manera mayoritaria, los estudios primarios analizados por las RS y MA conformaron sus muestras agrupando víctimas de distintas formas abusivas, de diversos tipos de vínculos con sus agresores y de abusos de duración variada. Esto compromete la validez de sus conclusiones las que se encuentran sesgadas por un error metodológico derivado de la insuficiente consideración de las distinciones fenomenológicas del ASI en la conformación de sus grupos en estudio. 
La variable evolutiva y su relación con el daño

La mirada sobre el desarrollo psicológico conlleva implicancias para el uso de las PP en la población de víctimas de ASI y su investigación. Por un lado, la edad de la víctima ha sido considerada como una variable relevante en relación a los efectos del ASI. Existe evidencia que da cuenta de la manifestación diferencial de síntomas en las víctimas de abuso sexual según su edad (Manly, Kim, Rogosch, \& Cicchetti, 2001 citado en Ramírez \& Fernández, 2011), de modo que esta variable sería uno de los factores que media el impacto del abuso en la víctima. Esto impactaría el tipo de indicador proyectivo que sería esperable según la etapa de desarrollo de la víctima.

Por otro lado, la posibilidad de uso de las distintas PP se ve determinada por las características que el desarrollo de los NNA presenta. Por ejemplo, las PP gráficas dependen del desarrollo de la función gráfica (dibujo), mientras que el uso de pruebas narrativas es dependiente del desarrollo de la capacidad narrativa. Por lo tanto, la indicación del uso de determinadas pruebas y su combinación dependerá, entre otros, de la edad del NNA.

Pese a lo anterior, tanto los estudios primarios como los secundarios sobre PP y ASI han tendido a no hacer distinciones evolutivas. Por ejemplo, en la RS realizada por Allen y Tussey (2012), que buscaba analizar si las PP gráficas podían detectar abuso sexual o físico, no se informa las edades de los sujetos muestrales de los estudios incluidos en la revisión, por lo que dicha variable no se incluyó en el análisis. Por otro lado, parte importante de los estudios primarios incluyen rangos etarios amplios en sus muestras (ver Apéndice A). Un total de 12 (57\%) de los 21 estudios de los cuales se reporta la edad de los sujetos de la muestra, incluyen edades que abarcan un rango de seis años o más, llegando a catorce años en el estudio de Cohen y Phelps (1985, citado en Trowbridge, 1995) que incluyó niños de entre 4 a 18 años. De solo uno de estos trabajos se reportan resultados de análisis por edad; Zivney, Nash y Hulsey (1988, citado en West, 1998) informaron respuestas diferenciales al test de Rorschach en víctimas de ASI de distintas edades.

La desconsideración de la variable edad supone que el desarrollo psicológico no afectaría la identificación de indicadores proyectivos. En otras palabras, se asume igual validez (o no validez) de los indicadores para todas las edades. Por otro lado, la composición muestral de los diseños reportados impide evidenciar la particularidad de grupos etarios específicos como los pre-escolares y los adolescentes. A la luz de los argumentos aquí planteados, esta aproximación teórica y metodológica podría estar dando lugar a conocimiento evolutivamente no pertinente.

Lo aquí planteado demuestra la existencia de una brecha entre el conocimiento sobre desarrollo evolutivo, fenomenología y psicopatología del ASI y los diseños metodológicos utilizados por los estudios empíricos sobre PP y ASI.

\section{Segunda brecha}

Las pruebas proyectivas como herramientas de evaluación psicológica: distancia entre la teoría psicodiagnóstica y la investigación

Las PP como instrumentos psicodiagnósticos se basan en el método clínico, desde teorías ideográficas de la personalidad (para una revisión crítica sobre el estatus científico de las PP ver Lilienfeld, Wood, \& Garb, 2000; Sneiderman, 2011; Passalacqua \& Febbraio, 2013). Estas teorías versan sobre fenómenos únicos y singulares, no múltiples y repetidos como es el caso de las ciencias nomotéticas. Consecuente con esta noción, Sneiderman (2011) plantea que las técnicas proyectivas son un método que busca aprehender la subjetividad de una persona de un modo holista. Es por eso que las PP no se utilizan como instrumentos aislados, sino que se enmarcan dentro del proceso psicodiagnóstico que evalúa la personalidad en su conjunto.

El resultado de la aplicación de una PP se entiende como una proyección de elementos mentales y afectivos provocada por las características poco estructuradas del material presentado. Estos resultados no se evalúan en el sentido cuantitativo puro, sino que forman parte de un proceso de toma de decisiones que parte de un modelo teórico e intenta analizar un fenómeno concreto, que puede ser real o simbólico y que puede darse en un aspecto manifiesto y/o latente (Sneiderman, 2011). Este fenómeno se expresa en las PP a través de indicadores gráficos, formales, verbales y otros. 
Un indicador proyectivo es todo aquel observable que puede vincularse con una variable psíquica (Passalacqua \& Febbraio, 2013). Constituyen observableslas respuestas verbales a una técnica, las producciones gráficas y los juegos, así como un silencio, una pausa, una interrupción en el discurso o un olvido (Sneiderman, 2011). La relación entre indicador y variable psíquica no es, de acuerdo a Passalacqua y Febbraio (2013), una relación unívoca sino más bien probabilística que establece el clínico a modo de inferencia basado en sus conocimientos y experiencia clínica.

Las PP forman parte del proceso psicodiagnóstico, el que contiene tres elementos a considerar: la batería de pruebas, la observación sistematizada de la conducta y la constelación de indicadores. En primer lugar, el proceso psicodiagnóstico se basa en el uso de una batería de pruebas, es decir, un conjunto de tests cuyo análisis integrado permite conocer mejor y con mayor profundidad a una persona. La tarea del evaluador será una adecuada selección de las pruebas que compondrán la batería para cada caso, resguardando la complementariedad de la información obtenida, al mismo tiempo que minimizando posibles errores de sesgos que puedan ser atribuidos a cada instrumento por separado. La información así obtenida será sometida para su análisis a un proceso de triangulación que permita la integración de los resultados (Sneiderman, 2011). Este proceso incluirá el cotejo inter-tests, en el cual se analizan las convergencias, divergencias y las redundancias observadas. De este modo, los indicadores que se recolectan con las técnicas en forma aislada, adquieren valor en relación con los demás datos.

El segundo elemento del proceso psicodiagnósticoes la observación de la conducta a laprueba, la que aporta indicadores tan relevantes como el contenido verbal o gráfico. La conducta observable en la persona examinada durante la evaluación permite inferir procesos psíquicos internos (Boekholt, 1998). La observación de la conducta resulta especialmente relevante en la evaluación de niños en los cuales el lenguaje verbal puede ser aún incompleto y en adolescentes en los cuales hay interferencia del lenguaje verbal, ya sea por alteraciones cognitivas $o$ afectivas.
Por último, tanto en el análisis intra como inter pruebas, las PP permiten reconocer constelaciones de indicadores que facilitan discriminar diferentes cuadros clínicos o distintos tipos de personalidad. La noción de indicadores es indisociable del concepto de constelación de datos (Pérez, 2009). Es dicha configuración o constelación la que aporta comprensión a la totalidad de los datos, articulándolos entre sí. En otras palabras, los indicadores que se recolectan con las técnicas, no significan nada de forma aislada, sino que su valor depende de la interrelación con los demás datos. Si bien han sido descritas constelaciones específicas de indicadores para características de personalidad o trastornos psíquicos, como la constelación del suicidio descrita por Exner (Rovira, 1983) y los indicadores emocionales complementarios encontrados por Maganto y Garaigordobil (2011), no siempre se presentan los mismos indicadores en todos los casos, pues dependen de cada persona en particular y de su contexto específico. El especialista es el que debere conocer dichos indicadores y su significación en cuanto a presencia o ausencia de un determinado cuadro clínico (Martorell \& Gómez, 2010).

Estos tres elementos forman parte esencial del psicodiagnóstico en el ámbito clínico, constituyendo la base del análisis e interpretación de las PP en dicho contexto. Sin embargo, dichos componentes han sido recogidos solo parcialmente por la investigación con PP y muy escasamente por los estudios que han explorado su uso en la evaluación de victimas de ASI. Es así que $21(81 \%)$ de los 26 estudios analizados en las RS y MA (ver Apéndice A) reportan resultados de la aplicación de dos o menos pruebas, 19 de los cuales (73\%) usaron solo una PP,lo que revela que la noción de batería psicodiagnóstica no estuvo a la base de sus diseños. Lo mismo ocurre con el concepto de constelación de indicadores, el que no ha sido utilizado en los modelos de análisis y prueba de hipótesis, siendo éste reemplazado por una aproximación reduccionista que busca establecer la relación entre indicadores aislados y la ocurrencia del abuso. Por último, ninguno de los estudios reportados incluyó la observación sistemática de la conducta de los sujetos examinados, pese al alto valor informativo que es posible suponer tendría la observación de 
conducta en niños que han sufrido experiencias de abuso. Por el contrario, estas investigaciones les han atribuido interés únicamente a los indicadores proyectivos directos (gráficos o verbales). En el caso de los estudios con PP gráficas, la producción gráfica de los niños ha quedado disociada de las verbalizaciones que acompañan el dibujo, en la medida que esta información no ha sido recogida ni analizada perdiéndose su riqueza informativa.

La escasa consideración de los componentes centrales de la práctica psicodiagnóstica por parte de los estudios científicos sobre PP y ASI con lleva importantes implicancias. Primero, las PP están siendo estudiadas en este campo en una modalidad que se aleja considerablemente del real uso de estas pruebas en el ámbito clínico. Esto supone, por un lado, una limitada capacidad de los diseños de investigación para recoger la riqueza y complejidad del mundo interno de los sujetos investigados; y, por otro, una falta de validez ecológica de los resultados al ser éstos fruto de una utilización empobrecida de las técnicas. En otras palabras, la capacidad de estos estudios de responder a la pregunta sobre la utilidad de las PP para evaluar víctimas de ASI se encuentra seriamente comprometida por la brecha que presentan sus diseños respecto de principios básicos del psicodiagnóstico que abarcan la modalidad de aplicación de las pruebas, de recolección de datos y de modos de análisis.

Respecto de esto último, los estudios empíricos sobre ASI y PP han analizado sus resultados conforme a las exigencias de la psicometría, la que se sustenta en las ciencias nomotéticas y cuyo paradigma de base es el positivismo o postpositivismo. Esta aproximación supone un desajuste paradigmático al aplicarse a técnicas que se basan en teorías ideográficas, cuya base paradigmática es psicoanalítica, fenomenológica o constructivista, como es el caso de las PP. Este desajuste se manifiesta en que, al realizar el análisis de datos, se aplican técnicas de análisis cuantitativo de tipo psicométrico, sin considerarla naturaleza cualitativa de la información entregada por las PP. Las autoras de este artículo no rechazamos el que se utilicen métodos cuantitativos para responder a la pregunta de investigación, sino que sostenemos que es necesario que éstos se ajusten al carácter de los datos arrojados por las PP de manera de no traicionar la esencia paradigmática que yace detrás de éstos.

Para ejemplificar esta postura, se comentará la polémica surgida entre West (2000) y Garb et al. (2000a) quienes realizaron los dos MA existentes sobre la validez del uso de las PP en víctimas de ASI. West $(1998,2000)$ consideró en la presentación de los resultados de su MA sólo aquellos indicadores que resultaron significativos para diferenciar a los sujetos con y sin abuso. Posteriormente, Garb et al. (2000b) reanalizaron los datos utilizados por West incluyendo en su análisis todos los indicadores que se habían seleccionado en los diferentes estudios incluidos en el MA, y los trataron como si fuesen ítems de un cuestionario, es decir, con la exigencia de que todos y cada uno de ellos aporten a la medición de la variable de interés, tal como lo establece la psicometría. Ciertamente, obtuvieron resultados muy diferentes, lo que alimentó la polémica. Frente a ésta, compartimos la postura de West en la medida que lo que interesa es identificar un grupo de indicadores dentro de un conjunto que contribuyan a diferenciardos poblaciones de niños (con y sin experiencias de abuso), sin esperar que todos los indicadores que se seleccionan para los estudios, deban necesariamente resultar significativos para concluir si las pruebas proyectivas aportan a este fin.

También resulta oportuno señalar que, en algunas de las investigaciones analizadas en las revisiones publicadas, se encontró indicadores significativos pero con baja frecuencia de casos, como el dibujo de genitales en el grupo de niños con abuso informado en la RS de Allen y Tussey (2012). Estos investigadores recomiendan desestimar ese indicador, decisión que no compartimos porque es sabido que no todos los niños que han sufrido abuso responden de manera similar, lo que implica que en algunos sujetos el indicador puede estar presente y en otros ausente. $\mathrm{El}$ hecho que estos indicadores presenten significación, aun cuando la sensitividad o el tamaño del efecto resulten bajos, nos parece suficiente para considerarlos como parte del conjunto de indicadores que usen los estudios empíricos que tengan como propósito conocer la utilidad de las PP en la evaluación de las víctimas de ASI. 
En este trabajo proponemos que, para avanzar en el desarrollo de una línea de investigación científica cuyo propósito sea esclarecer la validez de la PP en la evaluación de víctimas de ASI, es necesario que los elementos que conforman la práctica del psicodiagnóstico sean incorporados en aspectos específicos del diseño de investigación de dichos estudios y que éste se ajuste a la naturaleza de las PP como instrumentos psicodiagnósticos.

\section{Tercera brecha}

\section{Sesgos y deficiencias metodológicas en la investigación cuantitativa sobre PP y ASI}

Los criterios científicos para lainvestigación en Psicología han sido sistematizados en las recomendaciones de la American Psychology Association (APA) sobre práctica basada en la evidencia. En 2006, la APA definió este término para significar "la integración de la mejor investigación disponible con la experticia clínica" (APA 2006, pág. 273). Esta noción demanda que los psicólogos reconozcan las fortalezas y limitaciones de la evidencia obtenida en los distintos tipos de investigación. Al respecto, el grupo de trabajo Journal Article Reporting Standards (2008) enfatizó la importancia de que los investigadores provean más detalles en los informes de sus investigaciones, explicitando cómo fue conducida la investigación y qué se encontró (APA, 2008). Esto permite la reproductibilidad de la investigación, condición que junto al falsacionismo establecen los criterios esenciales y nucleares que determinan la validez de los resultados de las investigaciones.

La validez de los resultados de un estudio está estrechamente vinculada al diseño de la investigación. Se podrá lograr validez interna si se controla la influencia de otras variables extrañas, por ejemplo, seleccionando grupos de comparación homólogos en cuanto a las características que no están siendo investigadas, utilizando evaluadores ciegos a la pertenencia a gruposy que alcancen alta confiabilidad interevaluadores, definiendo operacionalmente las variables en estudio, estandarizando la aplicación de los instrumentos, utilizando criterios de inclusión y exclusión de la muestra, entre otros. Por su parte, la validez externa se logrará si los resultados obtenidos se aplican a poblaciones de características similares a las del estudio original. Resulta conveniente también replicar los estudios en poblaciones con características diferentes con el fin deconocer el alcance de los resultados. Las decisiones metodológicas que se orienten a favorecer la validez interna y externa de los estudios asegurarán la obtención de un conocimiento de alta calidad científica. Por el contrario, los estudios que presenten errores en su diseño podrán estar produciendo sesgos que comprometen su validez.

Respecto de la investigación sobre el uso de PP en la evaluación de víctimas de ASI, gran parte de ésta ha adolecido de fallas metodológicas (ver Apéndice B para un resumen de estas fallas). Estas debilidades han sido identificadas por las revisiones publicadas en este campo, las que han puesto en duda la calidad de los hallazgos de los estudios primarios y su interpretación. Dichas falencias han sido identificadas en cuestiones centrales de todo diseño, como la definición de variables, la conformación muestral, los procedimientos de aplicación de las pruebas y el tratamiento de los datos.

En primer lugar, las RS reportan una ausencia de definición operacional de las variables en estudio en los trabajos revisados. Asimismo, en relación al procedimiento, Trowbridge (1995) detectó diversos problemas en 6 de los 12 estudios sobre PP gráficas revisadas, como la no utilización de técnicas estandarizadas para recoger los datos, el uso de dibujos demasiado complejos para niños pequeños y el no reporte de materiales y consignas para la ejecución de dibujos o los sistemas de puntuación utilizados. Estas falencias no permiten la réplica de la investigación y comprometen la validez de los análisis.

En cuanto a la tabulación de la información, la literatura también ha criticado que, en gran parte de los estudios primarios, los evaluadores no fuesen ciegos a la hipótesis y a la pertenencia de los sujetos a los grupos en comparación (Trowbridge, 1995). Esta condición que fue cumplida solo por cinco (20\%) de los 25 estudios analizados por las revisiones, mientras que el $80 \%$ de los estudios no usaron evaluadores ciegos o fallaron en informar este aspecto del diseño. Respecto del análisis de datos, la confiabilidad inter-evaluadores constituye otro aspecto problemático identificado. En el caso de los 
trabajos revisados por Trowbridge (1995), el cálculo de coeficiente de confiabilidad entre los distintos evaluadores no se realizó en cuatro de ellos (33\%), mientras que West (1998) informó de este cálculo en solo tres de los estudios (25\%) que incluyó su MA. Por su parte, en la RS realizada por Allen y Tussey (2012), tres de los estudios (23\%) no examinaron el acuerdo entre los evaluadores, mientras que de los trabajos que realizaron este cálculo, ninguno de los indicadores individuales ni sistemas de puntajes examinados informó valores sobre .80. Esto sugiere que los resultados de los estudios primarios analizados en estos trabajos pueden haber dependido en gran cantidad del juicio subjetivo de quienes interpretaron los dibujos.

Por último, las RS y MA reportan insuficiencias en el muestreo utilizado por gran parte de los estudios primarios. Estas falencias se refieren al trabajo con muestras muy pequeñas (13 de los 25 estudios primarios, un 52\%, trabajaron con muestras de víctimas de ASI de $\mathrm{N}$ menor a 30), falta de información acerca de cómo se llevó a cabo el proceso de muestreo, o bien errores como el uso del autorreporte para establecer la existencia de abuso. Sin embargo, una deficiencia de muestreo mayor es la falta de inclusión de grupos de control clínicos. Al respecto, solo un $36 \%$ de los estudios analizados por las RS y MA (nueve estudios) compararon los resultados del grupo de NNA con abuso con una muestra clínica de NNA sin antecedentes de abuso y dos estudios no incluyeron muestra control. Esta falencia en la conformación de las muestras no permiten aclarar si los indicadores o sistemas de puntajes que se han estudiado son característicos de ASI o si pueden también encontrarse en otro tipo de trastornos psicológicos. En otras palabras, la especificidad de los indicadores proyectivos no ha sido claramente establecida, en la medida en que no se ha probado si éstos dan cuenta de una posible experiencia (abuso) o si más bien reflejan el proceso psicopatológico (síntoma), no diferenciándose éste último de otras posibles causas.

No solo los estudios primarios han sido objeto de críticas metodológicas, lo mismo ha ocurrido con los MA publicados. Al respecto, Allen y Tussey (2012) dan cuenta de una falta de prolijidad de los dos MA existentes en la inclusión y comparación de estudios primarios de distinta naturaleza. Los autores apuntan a que los trabajos tanto de West (1998) como de Garb et al. (2000b), no examinaron el tipo de test utilizado o el sistema de puntaje empleado por los distintos estudios incluidos en sus respectivas revisiones, sino que, por el contrario, agruparon los hallazgos de todos los estudios juntos. Así, por ejemplo, estos MA incluyeron estudios que usaron el Test de Rorschach, distintas pruebas gráficas, narrativas y sistemas de puntuación. Esta falta de discriminación, no permite concluir sobre la validez particular de pruebas de distinta naturaleza.

Resumiendo los argumentos planteados en este apartado, la investigación científica hasta ahora desarrollada con el fin de esclarecer la utilidad de las PP en la evaluación psicológica de NNA víctimas de abuso, presenta importantes falencias. Estas falencias representan las brechas existentes entre los diseños de investigación utilizados y el saber científico sobre el fenómeno del ASI, los principios del psicodiagnóstico, y los estándares metodológicos de la investigación científica. La identificación de las debilidades de la investigación en este campo, permite delinear los estándares necesarios para el desarrollo futuro de estudios empíricos de calidad. A continuación, se presenta una propuesta que sistematiza dichos estándares.

\section{Propuesta metodológica para una investigación cuantitativa de calidad}

En función de los criterios antes presentados y las críticas formuladas a los estudios publicados, presentamos una propuesta de estándares para la generación de evidencia sobre el uso de PP en la evaluación psicológica de NNA víctimas de ASI. Esta propuesta consiste en la sistematización de un conjunto de criterios para el desarrollo de estudios empíricos realizados con metodología cuantitativa en relación al uso de PP para la evaluación de esta población. Estos criterios se fundamentan en las exigencias psicométricas establecidas para los instrumentos psicológicos y que constituyen las bases de una investigación cuantitativa de calidad. La propuesta se estructura en tres niveles de análisis: teórico-conceptual, metodológico y estadístico-analítico. 


\section{Nivel teórico-conceptual}

En este nivel se requiere definir las variables consideradas en el estudio, salvaguardando la validez de constructo, es decir, evitando cometer errores al inferir sobre los constructos que caracterizan las operaciones del estudio.

\section{Variables}

La propuesta abarca sólo el nivel observacional que ofrecen las PP, es decir, los indicadores proyectivos como variables en estudio o variables dependientes, sin considerar ni el nivel interpretativo ni el diagnóstico (Passalacqua \& Febbraio, 2013). En el caso de las pruebas gráficas, las variables en estudio serán indicadores de tipo gráfico y para las pruebas narrativas indicadores formales y de contenido. En todos los casos es preciso realizar una clara definición conceptual y operacional de indicadores, sin olvidar considerar el lenguaje no verbal sistematizado en forma de variables o indicadores conductuales.

Es necesario también, establecer con claridad los valores que asume cada variable, así como el uso de criterios de puntuación objetivos y operacionalmente definidos. Por último, se debe establecer el carácter de las variables que, para el diseño de comparación de grupos, pueden ser variables dependientes, independientes, de control, moderadoras, etc. Para la conformación de la muestra de niños con ASI, es necesario considerar al menos la edad y sexo de la víctima, así como variables fenomenológicas relevantes tales como tipo y frecuencia del abuso y vínculo con el agresor. Es deseable también considerar otras variables fenomenológicas como medios utilizados por el agresor, reacción del entorno, y antecedentes de polivictimización, así como otras variables que pudiesen afectar los resultados como la edad de inicio del abuso, el tiempo transcurrido, existencia de terapia, entre otras.

\section{Instrumentos}

El hacer investigación con criterio cientificista respetando el carácter de las PP, obliga a reconocer lo que desde lo nomotético puede exigirse a éstas. $\mathrm{Al}$ respecto, es preciso analizar cuáles de las características psicométricas de los instrumentos (confiabilidad, validez y construcción de normas) pueden constituir objetivos y procedimientos metodológicos consecuentes.

Respecto de la confiabilidad, considerando el carácter ideográfico de las PP, la única forma recomendada para evaluarla, es la fiabilidad interjueces en que una muestra de protocolos es puntuada independientemente por dos o más evaluadores comparándose sus registros a través de correlaciones o coeficientes de confiabilidad (Martínez, Hernández-Lloreda, \& HernándezLloreda, 2006). No se recomienda calcular confiabilidad test re-test debido a que las PP, aun cuando evalúan aspectos estructurales de la personalidad, son sensibles a elementos situacionales. Por otra parte, el índice deconfiabilidad a través de consistencia interna, que alude al grado en que los elementos que conforman una prueba son homogéneos, no se espera que arroje valores altos debido a que los estímulos en el caso de pruebas temáticas o los indicadores en el caso de pruebas gráficas, exploran diferentes aspectos de la personalidad. Esta razón también es aplicable para no recomendar el cálculo de la fiabilidad por mitades.

De cualquier manera, se recuerda la relevancia de trabajar con una batería de pruebas proyectivas, cuya elección será acorde a los objetivos del estudio.

\section{Nivel técnico metodológico}

Este nivel se refiere al diseño metodológico que determina la validez tanto interna como externa. Defectos en este nivel pueden conducir a errores al inferir sobre la relación entre las variables o sobre la mantención de los resultados al variar los sujetos, los contextos, etc. Por esto, es altamente relevante el control de variables extrañas y las características de las muestras.

\section{Tipo de diseño (grupos)}

Se propone un diseño no experimental transversal de comparación de grupos con atribución de causalidad. La variable independiente (experiencia de abuso) ya ha ocurrido y se definen variables dependientes (indicadores gráficos, de contenido, formales, conductuales) que pueden considerarse la consecuencia de la variable independiente o, más precisamente, la manifestación de su impacto. La elección de los sujetos de los grupos de 
comparación no puede hacerse al azar, lo que determina que se controlen o al menos se reconozcan variables que pudieran incidir sobre $\mathrm{la}(\mathrm{s})$ variable(s) dependiente(s) en estudio.

La comparación de un grupo de niños con experiencias de abuso y grupos controles podrá esclarecer la manera en que se evidencia, o no, el impacto del abuso. Se recomienda utilizar grupos controles de niños con perturbación emocional o clínicos y muestras no clínicas de niños sin experiencias de abuso.

Por último, se recomienda trabajar con grupos de niños con ASI en que no haya superposición con otras condiciones estresantes $\mathrm{u}$ otras patologías. Especial consideración debe realizarse respecto del maltrato infantil, puesto que la identificación de las diferencias o similitudes del impacto de estas dos formas de violencia en las víctimas es un campo de investigación aún incipiente (por ej., Lewis, McElroy, Harlaar, \& Runyan, 2016).

\section{Muestra (características)}

La selección de muestras con características homólogas, y el estudio de las diferencias que esas características (por ej., sexo, edad) puedan arrojar, constituye uno de los objetivos de investigación en el caso de evaluar NNA con experiencias de ASI.

Es un requisito que los criterios de inclusión y exclusión estén claramente definidos, así como informar los criterios y procedimientos para la selección de las muestras. Se recomienda utilizar grupos de sujetos pareados y considerar la ausencia de otros desórdenes mentales tales como retardo mental, psicosis, o problemáticas confundentes (por ej. pérdidas o trauma reciente).

La selección de los sujetos que conformen la muestra de NNA con abuso, debe considerar el uso de pruebas externas de confirmación, tales como la existencia de evidencia médico legal, embarazo de la víctima como resultado del abuso, confesión del autor, declaración incriminatoria de testigos y/o existencia de sentencia judicial (Navarro, 2011).

La selección de sujetos para las muestras de NNA sin experiencias de abuso debiera incluir entrevista a los padres, a los profesores y antecedentes médico-psicológicos, con el fin de descartar a través de procedimientos sistemáticos la existencia de ASI en este grupo. Asimismo, la muestra clínica debe estar avalada por informes de profesionales idóneos.

Por último, el tamaño de las muestras debe ser el mayor posible, idealmente superar los 30 casos por grupo (Martínez et al., 2006).

\section{Procedimiento}

Se sugiere utilizar múltiples evaluadores para evitar sesgos en la decisión acerca de la existencia o características de un indicador. Es imprescindible realizar capacitaciones a los evaluadores para homologar criterios tanto para la toma de muestra como para el análisis.

Los evaluadores deben ser ciegos respecto de la proveniencia de grupos y se debe calcular la confiabilidad inter-evaluadores. También se debe informar cómo se resuelven los desacuerdos entre los evaluadores.

Asimismo, es necesario estandarizar el procedimiento de aplicación de los instrumentos e informar las consignas con el fin de que puedan ser replicadas.

\section{Nivel estadístico-analítico}

Este tercer nivel refiere a los resultados y su interpretación. Se busca resguardar la validez estadística, de modo que las conclusiones que se extraigan no sean equivocas o incorrectas.

\section{Tratamiento de los datos}

Es conveniente informar la significación estadística y práctica (tamaño del efecto) para tener una mayor certeza de las diferencias que se puedan encontrar entre los grupos en comparación.

$\mathrm{Al}$ evaluar indicadores o sistemas de puntajes, es decir datos categóricos, resulta adecuada la utilización del test estadístico de chi cuadrado y/o de Fisher los que permiten informar la probabilidad asociada al rechazo de una hipótesis nula verdadera. No obstante, con los valores de chi cuadrado se puede calcular e informar también el tamaño del efecto. Es decir que las tablas de contingencia permiten además informar la sensibilidad y calcular la razón de probabilidades.

Los estudios que utilicen sistemas de puntajes pueden aplicar tests o pruebas para datos medidos a nivel intervalar, lo que permite aplicar análisis de varianza, regresión lineal, t de student, análisis 
discriminante, entre otros, y los consecuentes cálculos de tamaño del efecto, entre los que se puede citar la d de Cohen., delta de Glass, g de Hegdes y eta al cuadrado.

Un modelo posible de análisis estadístico para la detección de constelación de indicadores consiste en seleccionar aquellos indicadores que han resultado con significación estadística sin exigir que presenten significación práctica (tamaños de efecto de moderados a altos). Una vez reconocidos, se puede aplicar una técnica de análisis multivariado, por ejemplo, análisis discriminante, que identifique cuáles indicadores permiten diferenciar a los grupos de NNA con abuso versus sin abuso y clínicos. De este modo, se podría llegar a establecer un listado de indicadores que no necesariamente debiera presentarse en todos los casos que han sufrido abuso, debido a la ya mencionada heterogeneidad del fenómeno y sus consecuencias. A través de sucesivas investigaciones se podrían establecer patrones diferenciales de los porcentajes de indicadores presentes en subgrupos específicos de NNA víctimas de abuso (por ej. abuso crónico, de evento único o abuso incestuoso), o en subgrupos según características de las víctimas como la edad y sexo.

Al respecto, el Equipo de Investigación sobre Psicodiagnóstico y Violencia Sexual de la Universidad de Chile ha venido sistemáticamente estudiando la presentación de los indicadores proyectivos de distintas PP en protocolos de víctimas de ASI de distintas edades (ver listado de estas investigaciones en Apéndice C). En conjunto estos estudios apuntan a la existencia de cambios en los patrones de respuesta de las víctimas frente a PP gráficas y narrativas a lo largo del ciclo evolutivo, siendo la etapa pre-escolar particularmente sensible al efecto de la variable evolutiva, debido en parte a la dificultad de los más pequeños para expresarse verbalmente y a la inhibición o funcionamiento regresivo que pueden presentar. Estos estudios avalan la necesidad de avanzar hacia la identificación de constelaciones de indicadores por rango etario.

La propuesta metodológica aquí formulada pretende acercar la investigación en el campo de la evaluación de víctimas de ASI a través del uso de PP al conocimiento científico disponible y a los estándares de cientificidad. Ajustándose a un marco cuantitativo de análisis, la propuesta provee un modelo operacional que permite la inclusión de nociones complejas, como la de batería psicodiagnóstica y constelaciones de indicadores, al mismo tiempo que ofrece orientaciones concretas para la inclusión de variables individuales y fenomenológicas relevantes que doten de pertinencia los diseños a utilizar. Ofrece así, un modelo metodológico operativo para la generación de evidencia empírica de calidad que responda a la pregunta sobre la validez de estos instrumentos para la evaluación de NNA que han sufrido abuso sexual.

\section{Discusión o consideraciones finales.}

Aunque el uso de las PP para la evaluación de víctimas de ASI es extendido en el ámbito clínico $\mathrm{y}$ forense, como hemos demostrado en este trabajo, la pregunta sobre la validez de estos instrumentos en la evaluación de esta población no ha sido abordada satisfactoriamente hasta ahora. Hemos argumentado que esto se debe a que los estudios publicados no han considerado de manera suficiente el carácter complejo del fenómeno del ASI y sus consecuencias, la naturaleza de las PP como instrumentos psicodiagnósticos ni los principios de cientificidad que deben guiar la investigación científica.

Hemos aquí presentado una propuesta para el diseño de futuras investigaciones destinadas a establecer el aporte y la validez de las PP en la evaluación de NNA víctimas de violencia sexual. Esta propuesta ofrece un modo operacional para considerar las variables relevantes que la literatura en este campo ha establecido median el impacto del abuso en las víctimas, así como las características del desarrollo de los sujetos en el diseño de la investigación. La propuesta también plantea un modelo integrativo y coherente que respeta la naturaleza de las PP como instrumentos cualitativos de evaluación, ciñéndose al mismo tiempo a los estándares o exigencias de la metodología cuantitativa.

En nuestra propuesta hemos también buscado ampliar el foco de la investigación sobre los instrumentos proyectivos en la población de víctimas de ASI, de modo que su estudio se acerque al potencial de las PP como instrumentos de evaluación. Así, el concepto de batería psicodiagnóstica, el reemplazo de la noción de 
indicador proyectivo por el concepto de constelación de indicadores, así como la inclusión de la observación conductual, son algunos de los elementos centrales e innovadores del modelo propuesto.

Sin dudas, el modelo de investigación que se propone representa desafíos para su implementación. Mayores y más precisas distinciones siempre se traducirán en diseños de investigación de mayor complejidad (por ej., más variables en estudio, muestras mayores), lo que resulta desafiante, por ejemplo, a la hora conformar muestras representativas equiparables de distintos rangos etarios. Sin embargo, es la propia naturaleza del objeto de estudio y de los instrumentos de evaluación la que exige esta complejidad metodológica. Cualquier aproximación cuyo diseño evada dicha complejidad, supone un reduccionismo que amenaza la posibilidad de arribar a conclusiones válidas y pertinentes.

En Latinoamérica, un elemento adicional que puede estar favoreciendo la desconsideración de los aspectos aquí mencionados, es la escasez de estudios normativos locales en población general que permitan evidenciar respuestas típicas y diferenciadas por edad a las distintas PP. Este es un desafío adicional que enfrenta el desarrollo científico en esta parte del mundo, con el fin de generar evidencia no solo científicamente avalada, sino que culturalmente pertinente.

En el campo de la investigación del impacto del ASI en las víctimas, queda aún por esclarecer el efecto mediador diferencial y relativo de las variables individuales y fenomenológicas discutidas en este trabajo. Asimismo, hay otros factores involucrados que requieren ser abordados, tales como los efectos del abuso según el sexo del agresor, su impacto en el desarrollo sexual y en otras esferas de la personalidad, así como el efecto del abuso en grupos específicos (por ej. en niños con discapacidad física o mental), por mencionar algunos. Por otro lado, la investigación sobre el tratamiento de las víctimas y los procesos de reparación del daño, requiere aún de mayor desarrollo. En todos esto ámbitos, las PP pueden ser herramientas de gran valor. Para que su uso sea válido será preciso desarrollar una línea de investigación comparable a partir de la cual diversos estudios empíricos puedan aportar evidencia acumulativa sobre la validez de distintas constelaciones de indicadores proyectivos provenientes de la aplicación de diferentes PP. El conocimiento así producido ofrecería a los profesionales de la psicología clínica y forense un marco de evidencia empírica que establezca los límites y alcances de estos instrumentos como herramientas para la detección y evaluación del ASI. Esperamos quela propuesta aquí formulada contribuya al desarrollo de ese horizonte.

\section{Referencias $^{2}$}

Adler-Nevo, G., \& Manassis, K. (2005). Psychosocial treatment of pediatric posttraumatic stress disorder: The neglected field of single-incident trauma. Depression and Anxiety, 22(4), 177-189.

*Allen B., \& Tussey Ch. (2012). Can projective drawings detect if a child experienced sexual or physical abuse? A systematic review with the controlled research. Trauma, Violence \& Abuse, 13(2), 97-111.

APA Presidential Task Force on Evidence-Based Practice. (2006). Evidence-based practice in psychology. The American Psychologist, 61(4), 271.

APA Publications and Comunicaions Board Working Group on Journal Article Reporting Standards (2008). Reporting Standards for Research in Psychology. Why do weneedthem? What might theybe? American Psychologist, 63(9), 839-851.

Beigbeder, C., Balarini, Z., \& Colombo, R. I. (2005). Abuso y maltrato infantil: Inventario de frases revisado (IFR). Buenos Aires: Cauquen Editora.

Boekholt M. (1998). Epreuves thématiques en clinique infantile. Paris: Dunod

Chen, L. P., Murad, M. H., Paras, M. L., Colbenson, K. M., Sattler, A. L., Goranson, E. N., ...,\& Zirakzadeh, A. (2010). Sexual abuse and lifetime diagnosis of psychiatric disorders: Systematic review and metaanalysis. Mayo Clinic Proceedings, 85(7), 618-629.

\footnotetext{
${ }^{2}$ Las referencias marcadas con asterisco indican estudios incluidos en la revisión.
} 
Collin-Vézina, D., Daigneault, I., \& Hébert, M. (2013). Lessons learned from child sexual abuse research: Prevalence, outcomes, and preventive strategies. Child and Adolescent Psychiatry and Mental Health, 7(1), 22.

Colombo, R., \& Beigbeder, C. (2003) Abuso y maltrato infantil. Hora de Juego Diagnóstica. Santiago del Estero, Capital Federal, Argentina: Sainte Claire Editora.

Finkelhor, D., \& Browne, A. (1985). The traumatic impact of child sexual abuse: A conceptualization. American Journal of Orthopsychiatry, 55(4), 530-541.

Foster, J. M., \& Carson, D. K. (2013). Child sexual abuse in the United States: Perspectives on assessment and intervention. American Journal of Humanities and Social Sciences, 1(3), 97-108.

Furlan, A. D., Pennick, V., Bombardier, C., \& van Tulder, M. (2009). 2009 updated method guidelines for systematic reviews in the Cochrane Back Review Group. Spine, 34(18), 1929-1941.

Garb, H. N., Wood, J. M., \& Nezworski, M. T. (2000a). Letter to the editor: Projective techniques and the detection of child sexual abuse. Child Abuse \& Neglect, 24, 437-438.

*Garb, H. N., Wood, J. M., \& Nezworski, T. M. (2000b). Projective techniques and the detection of child sexual abuse. Child Maltreatment Journal: Journal of the American Professional Society on the Abuse of Children, 5, 161- 168.

Hillberg, T., Hamilton-Giachritsis, C., \& Dixon, L. (2011). Review of meta-analyses on the association between child sexual abuse and adult mental health difficulties: A systematic Approach. Trauma, Violence \& Abuse I2(1), 38-49.

Huerta, S., Maric, V., \& Navarro, C. (2002). Factores que intervienen en el impacto del abuso incestuoso sobre la víctima. Ter. Psicol, 20(2), 117-124.

Kendall-Tackett, K. A., Williams, L. M., \& Finkelhor, D. (1993). Impact of sexual abuse on children: A review and synthesis of recent empirical studies. Psychological Bulletin, 113(1), 164-180.

Koppitz, E. (1980). El test guestáltico visomotor para niños. Buenos Aires: Guadalupe.
Lewis, T., McElroy, E., Harlaar, N., \& Runyan, D. (2016). Does the impact of child sexual abuse differ from maltreated but non-sexually abused children? A prospective examination of the impact of child sexual abuse on internalizing and externalizing behavior problems. Child Abuse \& Neglect, 51, 31-40.

Lilienfeld, S., Wood, J., \& Garb, H. (2000). The scientific Status of projective techniques. Psychological Science in the public interest. 2(1), 27-66.

Maganto, C., \& Garaigordobil, M. (2011). Indicadores emocionales complementarios para la evaluación emocional del Test del dibujo de dos figuras humanas (T2F). Revista Iberoamericana de Diagnóstico y Evaluación - e Avaliação Psicológica, 31(1), 73-95.

Marriott, C., Hamilton-Giachritsis, C., \& Harrop, C. (2014). Factors promoting resilience following childhood sexual abuse: A structured, narrative review of the literature. Child Abuse Review, 23(1), 17-34.

Martínez Arias, R., Hernández-Lloreda, M. J., \& Hernández-Lloreda, M. V. (2006). Psicometría. Madrid: Alianza Editorial.

Martorell, C., \& Gómez, O. (2010). Enfoque de la Evaluación Psicológica de la Revista Iberoamericana de Revista Iberoamericana de Diagnóstico y Evaluación - e Avaliação Psicológica, 30(2), 35-56.

Navarro, C. (2011). Evaluación de la credibilidad discursiva de niños, niñas y adolescentes víctimas de agresiones sexuales. En $P$. Quintana, M.Heresi, M. I. Diez, G.Macurán, M. D. L.Harpe, F. Quiroga, ... \& M. I. Léniz. Investigaciones en psicología clínica infanto juvenil: primer compendio de tesis del Magister en Psicología, mención psicología clínica infanto juvenil. Santiago, Chile: Universidad de Chile.

Passalacqua, A. M., \& Febbraio, A. (2013). Acerca de la validez y confiabilidad de las técnicas proyectivas. En $V$ Congreso Internacional de Investigación y Práctica Profesional en Psicología XX Jornadas de Investigación Noveno Encuentro de Investigadores en Psicología del MERCOSUR. Buenos Aires: Facultad de Psicología-Universidad de Buenos Aires. 
Pérez Lalli, M. (2009). Indicador: Algunas reflexiones sobre las implicancias de esta noción en el campo del psicodiagnóstico. Revista de Epistemología y Ciencias Humanas, (2).

Ramírez, C., \& Fernández, A. (2011). Abuso sexual infantil: Una revisión con base en pruebas empíricas. Psicología Conductual, 19(1), 7.

Rovira, F. (1983). El Rorschach. Nuevas aportaciones de J. E. Exner al sistema comprensivo. Estudio sistemático y análisis de las variables. España: Siglo veintiuno editores.

Sawyer, G. K., \& Hansen, D. J. (2014). Heterogeneous symptom patterns of sexually abused children in treatment: Understanding the complexity of the problem. Journal of Child Sexual Abuse, 23(8), 900-917.

Sneiderman, S. (2011). Consideraciones acerca de la confiabilidad y validez en las técnicas proyectivas. Subjetividad y Procesos Cognitivos, 15(2), 93-110.

Scott, M. T., Manzanero, A. L., Muñoz, J. M., \& Köhnken, G. (2014). Admisibilidad en contextos forenses de indicadores clínicos para la detección del abuso sexual infantil. Anuario de Psicología Jurídica, 24(1), 57-63.

*Trowbridge, M. M. (1995). Graphic indicators of sexual abuse in children's drawings: A review of literature. Arts in Psychotherapy, 22, 485493.

*West, M. (1998). Meta-analysis of studies assessing the efficacy of projective techniques in discriminating child sexual abuse. Child Abuse \& Neglect, 22(11), 1151-1166.

West, M. (2000). Response to Garb et al.'s comments about meta-analysis of studies using projectives with child sexual abuse subjects. Child Abuse \& Neglect, 24(4), 439441. 


\section{Apéndice A}

Tabla A. Resumen de estudios empíricos incluidos en las RS y MA sobre PP y ASI

\begin{tabular}{|c|c|c|c|c|}
\hline Estudio* & Muestra & Técnica: Puntuación & Resultados significativos & Resultados no significativos \\
\hline $\begin{array}{l}\text { Allan y Crandall } \\
(1986) \\
\text { G W }\end{array}$ & $\begin{array}{l}\text { niños y niñas de } 9-10 \\
\text { años: } 10 \text { coping y } 10 \text { no- } \\
\text { coping ( } 5 \text { ASI, } 1 \\
\text { maltrato físico y } 1 \\
\text { negligencia) }\end{array}$ & $\begin{array}{l}\text { Estrategia de } \\
\text { Visualización de } \\
\text { Rosebush: } \\
\text { subescalas de } \\
\text { Elkisch (1960) }\end{array}$ & $\begin{array}{l}\text { Rigidez y } \\
\text { despreocupación, y otros } \\
\text { elementos cualitativos }\end{array}$ & $\begin{array}{l}\text { Dos de las subescalas de } \\
\text { Elkisch (1960) y perspectiva } \\
\text { de Rosebush }\end{array}$ \\
\hline $\begin{array}{l}\text { Blanchouin, } \\
\text { Olivier, } \\
\text { Lighezzolo y } \\
\text { Tychey }\end{array}$ & $\begin{array}{l}20 \text { sobrevivientes de } \\
\text { ASI; } 39 \text { control } \\
\text { normales }\end{array}$ & $\begin{array}{l}\text { H-T-P/DAP: Van } \\
\text { Hutton }\end{array}$ & $\begin{array}{l}\text { Escala: Preocupación con } \\
\text { conceptos sexuales } \\
\text { relevantes }\end{array}$ & $\begin{array}{l}\text { Tres escalas: Agresión y } \\
\text { hostilidad; Retirada y } \\
\text { accesibilidad vigilada; } \\
\text { Alerta para el peligro, }\end{array}$ \\
\hline $\begin{array}{l}(2005) \\
A\end{array}$ & $\begin{array}{l}20 \text { sobrevivientes de } \\
\text { ASI; } 39 \text { control } \\
\text { psiquiátricos }\end{array}$ & $\begin{array}{l}\text { H-T-P/DAP: Van } \\
\text { Hutton }\end{array}$ & $\begin{array}{l}\text { Escala: Preocupación con } \\
\text { conceptos sexuales } \\
\text { relevantes }\end{array}$ & $\begin{array}{l}\text { sospecha y falta de confianza } \\
\text { Tres escalas: Agresión y } \\
\text { hostilidad; Retirada y } \\
\text { accesibilidad vigilada; } \\
\text { Alerta para el peligro, } \\
\text { sospecha y falta de confianza }\end{array}$ \\
\hline $\begin{array}{l}\text { Burgess (1988) } \\
\mathrm{T}\end{array}$ & $\begin{array}{l}9 \text { sujetos identificados } \\
\text { como sexualmente } \\
\text { abusados y } 8 \text { sujetos no } \\
\text { abusados; promedio de } \\
\text { edad } 5,10 \text { años. }\end{array}$ & $\begin{array}{l}7 \text { dibujos (clima } \\
\text { favorito, yo ahora, } \\
\text { yo cuando más } \\
\text { pequeño, la familia } \\
\text { haciendo algo, el } \\
\text { evento, una casa y } \\
\text { un árbol, dibujo } \\
\text { libre): Análisis } \\
\text { descriptivo; } 54 \\
\text { características } \\
\text { gráficas }\end{array}$ & $\begin{array}{l}\text { Mayor fragmentación, } \\
\text { pobre integración de } \\
\text { partes del cuerpo, figuras } \\
\text { de menos de } 23 \text { cms., } \\
\text { sexualización y } \\
\text { sombreado de partes del } \\
\text { cuerpo, caras menos } \\
\text { felices y más tristes, } \\
\text { dibujo poco claro del } \\
\text { evento, entre otros }\end{array}$ & No reportado \\
\hline $\begin{array}{l}\text { Burgess, } \\
\text { Hartman, } \\
\text { Wolbert y Grant } \\
\text { (1987) } \\
\text { T }\end{array}$ & $\begin{array}{l}12 \text { niñas de } 6-9 \text { años } \\
\text { sexualmente abusadas } \\
\text { por chofer del transporte } \\
\text { escolar }\end{array}$ & $\begin{array}{l}7 \text { dibujos (clima } \\
\text { favorito, yo ahora, } \\
\text { yo cuando más } \\
\text { pequeño, la familia } \\
\text { haciendo algo, el } \\
\text { evento, una casa y } \\
\text { un árbol, dibujo } \\
\text { libre): Análisis } \\
\text { descriptivo; } 6 \\
\text { categorías }\end{array}$ & $\begin{array}{l}\text { Indicadores de ansiedad, } \\
\text { inseguridad, aislamiento }\end{array}$ & No reportado \\
\hline $\begin{array}{l}\text { Chantler, Pelco } \\
\text { y Mertin (1993) } \\
\text { A G T W }\end{array}$ & $\begin{array}{l}\text { Niños y niñas de } 6-12 \\
\text { años: } 26 \text { sobrevivientes } \\
\text { de ASI, } 37 \text { control } \\
\text { psiquiátricos, } 39 \text { control } \\
\text { normales }\end{array}$ & $\begin{array}{l}\text { HFD: Koppitz y } 7 \\
\text { items badera } \\
\text { (Koppitz, 1968) }\end{array}$ & $\begin{array}{l}\text { Puntaje total EI; Total de } \\
\text { EI ítems de bandera roja }\end{array}$ & Ninguno \\
\hline $\begin{array}{l}\text { Cohen y Phelps } \\
(1985) \\
\text { A T }\end{array}$ & $\begin{array}{l}\text { Estudio 1: } 89 \\
\text { sobrevivientes de ASI, } \\
77 \text { control psiquiátricos, } \\
\text { todos de } 4-18 \text { años } \\
\text { Estudio } 2: 40 \\
\text { sobrevivientes de ASI, } \\
40 \text { control psiquiátricos, } \\
\text { todos de } 4-18 \text { años }\end{array}$ & $\begin{array}{l}\text { H-T-P: } 12 \\
\text { indicadores; KFD: } \\
12 \text { indicators } \\
\text { Free Draw: } 12 \\
\text { indicators } \\
\text { H-T-P: } 12 \\
\text { indicadores }\end{array}$ & $\begin{array}{l}\text { Puntaje Total } \\
\text { Puntaje Total } \\
\text { Ninguno } \\
\text { Ninguno }\end{array}$ & $\begin{array}{l}\text { Ninguno } \\
\text { Ninguno } \\
\text { Puntaje Total } \\
\text { Puntaje Total }\end{array}$ \\
\hline $\begin{array}{l}\text { Hackbarth, } \\
\text { Murphy y } \\
\text { McQuary (1991) } \\
\text { A G T W }\end{array}$ & $\begin{array}{l}\text { Niños y niñas de } 6-13 \\
\text { años: } 30 \text { sobrevivientes } \\
\text { de ASI, } 30 \text { control } \\
\text { normales }\end{array}$ & KFD: LILIF & Bajo puntaje LILIF & $\begin{array}{l}\text { Acciones; figuras } \\
\text { características; posición, } \\
\text { distancia y barreras }\end{array}$ \\
\hline $\begin{array}{l}\text { Henderson } \\
(1990) \\
\text { G W }\end{array}$ & $\begin{array}{l}112 \text { niñas de } 4-16 \text { años: } \\
56 \text { con abuso sexual } \\
\text { ( } 56 \% \text { por parte de figura } \\
\text { paterna), } 56 \text { sin abuso }\end{array}$ & TAT: no reportado & $\begin{array}{l}\text { Actos desagradables, } \\
\text { Reciprocidad negativa, } \\
\text { No reciprocidad, Fallo de } \\
\text { afrontamiento }\end{array}$ & $\begin{array}{l}\text { Afecto negativo, No } \\
\text { afrontamiento, Referencia a } \\
\text { daño, nfermedad o muerte }\end{array}$ \\
\hline $\begin{array}{l}\text { Hibbard y } \\
\text { Hartman } \\
(1990 \text { a) } \\
\text { A T }\end{array}$ & $\begin{array}{l}\text { Niños y niñas de } 3-8 \\
\text { años: } 94 \text { supuestos } \\
\text { sobrevivientes de } \\
\text { ASI, } 100 \text { control } \\
\text { normales }\end{array}$ & $\begin{array}{l}\text { D-A-M: } 4 \\
\text { indicadores }\end{array}$ & Pene o vagina & $\begin{array}{l}\text { Pechos, Ombligos, Recto } \\
\text { Ira/agresión, Impulsividad, } \\
\text { Inseguridad/inadecuación, } \\
\text { Timidez }\end{array}$ \\
\hline $\begin{array}{l}\text { Hibbard y } \\
\text { Hartman } \\
(1990 b)\end{array}$ & $\begin{array}{l}\text { Niños y niñas de } 5-8 \\
\text { años: } 65 \text { supuestos } \\
\text { sobrevivientes de ASI, }\end{array}$ & HFD: Koppitz & Categoría EI: ansiedad & $\begin{array}{l}\text { Todos los EI, incluyendo: } \\
\text { genitales, longitud del brazo, } \\
\text { nubes, omisión de partes del }\end{array}$ \\
\hline
\end{tabular}




\section{A G T W 64 control normales}

Howe, Burgess
Hibbard, Roghmann y

Hoekelman

(1987)

A T

y McCormack

(1987)

A T

Kelley (1984)

$\mathrm{T}$

Niños y niñas de 3-7

años: 52 supuestos

sobrevivientes de ASI;

52 control normales

Adolescentes de 15-20 años: 12 sobrevivientes fugitivos de la AS, 24 controles de fugitivos

10 supuestas víctimas de ASI de 3-10 años

Leifer Shapiro,

Martone y

Kassem (1991)

G W

Louw y

Ramkisson (2002)

A

Ornduff et al. $\quad 42$ niñas de 5-16 años:

(1994)

G W

Palmer et al. (2000)

A

47sobrevivientes de ASI, 82 control normales sexualmente abusadas y sin abuso

23 niñas sobrevivientes de ASI, 17 niñas control normales

17 sexualmente abusadas, 25 grupo clínico sin historia de abuso
DAP: Cinco Ninguno

indicadores

DAP: 20 indicadores

Género menos ambiguo, calidad de línea débil / incompleta

\section{4 dibujos (autorretrato, el agresor, qué pasó y una persona completa): Análisis descriptivo usando criterio clínico \\ Rorschach: 9 variables Rorschach \\ H-T-P/DAP: Van Hutton}

TAT: Puntuación de las relaciones objetales y Sistema de puntuación de la cognición social

H-T-P: Van Hutton

\section{$20 \%$ presencia de} genitales en autroretrarto, $40 \%$ énfasis en la zona pélvica, $43 \%$ de énfasis en la zona baja en dibujos de niñas, $30 \%$ dibujos sin manos, entre otros WSUMSPC6, X-\%, D, ES, DEPI, Suma de Sombras, Ansiedad, Hostilidad, Penetración 4 escalas: Preocupación con conceptos sexuales relevantes; Agresión y hostilidad; Retirada y accesibilidad vigilada; Vigilancia por peligro, sospecha y falta de confianza

Complejidad de la representación de personas; Afecto-tono de la relación; Capacidad de inversión emocional en Rel. y normas morales; Comprensión de la causalidad social Ninguno cuerpo y tamaño de partes del cuerpo; Categorías IE: enojo, impulsividad, Inseguridad, timidez; Puntaje total

Pene, vagina, ojos, ano, ombligo

18 indicadores, incluyendo: completación de figuras, genitales, senos, omisión de partes del cuerpo, área genital, piernas juntas No reportado

F\%, EA, Zf, Lambda, Afr, Humanos, Índice de aislamiento, Barrera, Respuestas Ninguno

No reportado

Cinco escalas: Preocupación con conceptos sexuales relevantes; Agresión y hostilidad; Retirada y accesibilidad vigilada; Vigilancia por peligro, sospecha y falta de confianza; Puntaje Total Ninguno indicadores, dos gráfico-representativa, escalas

omisión de sujetos, distorsión corporal, proximidad emocional; Escalas: depresión, ansiedad

Preocupación Sexual, culpa puntuación para el TAT

Inferioridad, ansiedad, depresión, ira, hostilidad, resultados favorable, resultados no favorables

Afecto, Actividad,

Hand Test:

Rasch y Wagner 48 niñas de 6-17 años:

Variables Hand Test

Entorno, Desajuste, Respuestas, Repetición agresión, interpersonal,
Dependencia, comunicación, exhibición, dirección, adquisición, pasividad, tensión, minusvalía, miedo, descriptivo, bizarro, falla, retiro, patología, AI RT, H-L, AOR 
Sidun y

Rosenthal

(1987)

A T

30 sobrevivientes psiquiátricos de ASI, 3 control psiquiátricos, todos de 13-17 años

Stovall y Craig
(1990)
G W

60 niñas de $7-12$ en tres grupos: abuso físico, abuso sexual, grupo clínico sin historia de abuso

TAT: Escala de

Niñas de entre 3,5-17

Yates, Beutler Crago (1985)

A $\mathrm{T}$ años: 18 sobrevivientes de incesto, 17 control psiquiátricos

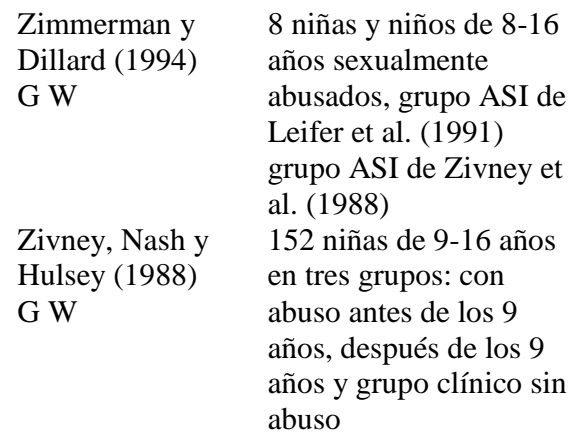
Relaciones Objetales Internalizadas (Taylor \& Franzen, 1986) y Escala de Aron (1949)

HFD: 15 indicadores

Indicadores: Manos omitidas, suficiente Integración corporal; Escala: Rasgos sexuales, pechos o formas en cuñas/fálico/círculo

Diferenciación yo-otro,

Diferenciación otro-otro,

Disposición del otro

hacia el yo, No contacto

con otros, Disposición

del yo hacia el yo,

Extensión de la interacción, Satisfacción de las relaciones, Naturaleza del vínculo yo-otro

Hipersexualización, inmadurez, sublimación

\section{Rorschach: Exner}

$\mathrm{X}-\%, \mathrm{~F} 1 \%$, D, EA, DEPI, Lambda, Afr, Barrera, Penetración

Rorschach :115
variables Rorschach

Diferencias en la patología grupo abuso temprano v/s abusos tardíos, con víctimas de abuso temprano mostrando
72 indicadores, entre ellos: figuras sobre-sexualizadas, genitales abiertos, mano que cubre la zona genital, ojos vacíos

5 escalas, incluyendo: sexualidad, ansiedad, baja autoestima

Las tres subescalas de Aron (1949)

Sobre-elaboración de características sexuales masculinas,

Sobre-elaboración de características sexuales femeninas

WSUMSPC6, ES, Suma de sombra, Zf, Figura humana pura, Índice de aislamiento, Respuestas funcionamiento psicológico más primitivo. Cinco variables Rorschach

Información extraída de Allen y Tussey (2012), Garb et al. (2000), Trowbridge (1995) y West (1998).

*Se identifica la revisión en que cada estudio primario fue incluido con la inicial del primer autor ( $A=$ Allen y Tussey, 2012; G= Garb et al., 2000; T= Trowbridge, 1995; y W= West, 1998).

Nota. Todos los hallazgos significativos indican que los grupos ASI obtuvieron puntuaciones significativamente más desadaptativas que el grupo control.

Nota . Afr = proporción de afectos; AI RT = promedio de tiempo de respuesta inicial; AOR = puntajes de acting out; D-A-M = Dibujo-un-Hombre; $\mathrm{DAP}=$ Dibujo-una-Persona; $\mathrm{EA}=$ experiencia real; $\mathrm{EI}=$ Indicadores Emocionales; $\mathrm{ES}=$ simulación experimentada; $\mathrm{F}+\%=$ forma pura; $\mathrm{HFD}=$ Dibujo de la Figura Humana; H-L = tiempo de respuesta alto menos bajo; H-T-P = Casa-Árbol-Persona; KFD = Dibujo Familia Kinética; LILIF = Procedimiento de calificación Like to Live in Family; TAT = Test de Apercepción Temática; WSUMSPC6 = sumatoria ponderada de los seis puntajes especiales; $\mathrm{Zf}=$ frecuencia de $\mathrm{Z}$. 


\section{Apéndice B}

Tabla B. Resumen de falencias metodológicas identificadas en los estudios primarios analizados en las RS y

\section{MA sobre PP y ASI}

\begin{tabular}{ll}
\hline Estudio & Problemas de procedimiento \\
\hline Allan y Crandall (1986) & \\
$\begin{array}{l}\text { Blanchouin, Olivier, } \\
\text { Lighezzolo y Tychey } \\
(2005)\end{array}$ & \\
Burgess (1988)* & $\begin{array}{l}\text { Uso de una serie de dibujos } \\
\text { que puede ser compleja para } \\
\text { niños pequeños }\end{array}$ \\
& $\begin{array}{l}\text { Falla en describir los medios } \\
\text { elegidos }\end{array}$ \\
$\begin{array}{l}\text { Falla en describir los medios } \\
\text { elegidos }\end{array}$ \\
$\begin{array}{l}\text { Wolbert y Grant } \\
\text { (1987)* }\end{array}$ & $\begin{array}{l}\text { que pue una serie de dibujos } \\
\text { niños pequeños }\end{array}$ \\
& $\begin{array}{l}\text { Características de calificación } \\
\text { pobremente definidas }\end{array}$
\end{tabular}

Chantler, Pelco y pobremente definidas

Mertin (1993)*

\section{Cohen y Phelps $(1985)^{*}$ \\ Hackbarth, Murphy y McQuary (1991)*}

Henderson (1990)

Hibbard y Hartman (1990a)*

Hibbard y Hartman (1990b)*

Hibbard, Roghmann y Hoekelman (1987)*

Howe, Burgess y

McCormack (1987)*

\section{Kelley (1984)*}

Leifer Shapiro,

Martone y Kassem

(1991)

Louw y Ramkisson

(2002)

Ornduff et al., 1994

Palmer et al. (2000)

Problemas con evaluadores

No reportan si usaron múltiples evaluadores y si fueron ciegos No informan confiabilidad interjueces

No usaron evaluadores fueron ciegos

Problemas de muestreo

Muestra pequeña

No incluyó muestra clínica

Muestra ASI pequeña

Poco claro si evaluadores fueron ciegos

No midieron confiabilidad interjueces

Pobre confiabilidad inter-jueces

Falla en la descripción completa de los medios utilizados por los sujetos Diferencias en la recopilación de datos

Falla en describir medios usados

Los investigadores informaron selectivamente únicamente resultados estadísticamente significativos

Falta de descripción clara de los resúmenes estadísticos Resultados presentados en una forma confusa

No reportan si evaluadores fueron ciegos

No reportan estadística sobre el nivel de confiabilidad inter-jueces No reportan si evaluadores fueron ciegos

No midieron confiabilidad interjueces

No reportan si evaluadores fueron ciegos

Proporcionaron una selección de tres medios de dibujo que pueden crear una variable de confusión

Uso de dos medios de dibujo Criterios de calificación pobremente descritos
No reportan si evaluadores fueron ciegos

No midieron confiabilidad interjueces

Solo un evaluador no ciego

No usaron evaluadores ciegos

No reportan si evaluadores fueron ciegos

Confiabilidad inter-jueces bajo a
Muestra pequeña

Métodos de muestreo mal descritos para el grupo control No incluyó muestra clínica

Muestra pequeña

Falta de grupos de comparación

Muestra ASI pequeña

No incluyó muestra clínica

No incluyó muestra clínica

No incluyó muestra clínica

No incluyó muestra clínica

No incluyó muestra clínica

Muestra ASI pequeña

Uso de auto-reporte para

determinar el abuso

No incluyó muestra clínica

Muestra pequeña

Falta de grupos de comparación

No incluyó muestra clínica

Muestra ASI pequeña

No incluyó muestra clínica

No incluyó muestra clínica 
Piperno, Di Biasi y

Levi (2007)

Pistole y Ornduff

(1994)

Rasch y Wagner (1989)

Sidun y Rosenthal (1987)*

Stovall y Craig (1990)

Yates, Beutler y Crago

(1985)*

Zimmerman y Dillard (1994)

Procedimientos no estandarizados de muestreo y de técnicas de recolección de datos, no descripción completa de los medios, características de clasificación descritas incompletamente.

Los resultados pueden haberse obtenido por azar

Recolección de datos no estandarizados

Falta de descripción de los medios usados

Criterios de calificación mal definidos moderada de todas las subescalas. Aparentemente, no usaron evaluadores ciegos No midieron confiabilidad interjueces No reportan si evaluadores fueron ciegos

No reportan si evaluadores fueron ciegos

No reportan si evaluadores fueron ciegos

Muestra ASI pequeña

Muestra ASI pequeña

Procedimientos de muestreo para sujetos insuficientemente descritos

Muestra ASI pequeña

No incluyó muestra clínica

Zivney, Nash y Hulsey (1988)

Información extraída de Allen y Tussey (2012), Garb et al. (2000), Trowbridge (1995) y West (1998).

Nota: Solo los 12 estudios primarios incluidos en la RS de Trowbridge (1995), identificados con asterisco (*), fueron analizados sistemáticamente respecto de la calidad metodológica de sus diseños. De los otros estudios, las revisiones no ofrecen información exhaustiva al respecto por lo que la tabla informa solo la información disponible.

\section{Apéndice C}

Tabla C. Estudios Equipo de Investigación sobre Psicodiagnóstico y Violencia Sexual de la Universidad de Chile*

\begin{tabular}{|c|c|c|}
\hline Autor & Año & Título \\
\hline Arenas C., y Zapata, V. & 2014 & $\begin{array}{l}\text { Estudio descriptivo comparativo: desarrollo evolutivo en la expresión gráfica en la prueba } \\
\text { persona bajo la lluvia, en niños víctimas de agresión sexual (Memoria de título). }\end{array}$ \\
\hline Blanco, A., y Rojas, M. & 2008 & $\begin{array}{l}\text { Estudio Exploratorio-Descriptivo-Comparativo de características de las expresiones } \\
\text { gráficas en la Prueba Persona Bajo la Lluvia, en adolescentes de } 11 \text { a } 13 \text { años víctimas de } \\
\text { agresión sexual }\end{array}$ \\
\hline Cabrera, $\mathrm{T}$. & 2016 & $\begin{array}{l}\text { Vínculos, imagen de sí mismo e imagen de mundo en niños y niñas de } 6 \text { a } 10 \text { años víctimas de } \\
\text { agresión sexual, a través del Test de Apercepción Temática Infantil con Figuras Animales } \\
\text { (CAT-A) (Memoria de título). }\end{array}$ \\
\hline $\begin{array}{l}\text { Giménez, G., y Pérez, } \\
\text { M. F. }\end{array}$ & 2014 & $\begin{array}{l}\text { Diferencias en las producciones gráficas de víctimas de agresión sexual infantil y víctimas de } \\
\text { maltrato físico infantil, en las pruebas Dibujo de la Figura Humana y Persona Bajo la Lluvia } \\
\text { (Memoria de título). }\end{array}$ \\
\hline Huerta, S. & 2013 & $\begin{array}{l}\text { Caracterización del funcionamiento psicológico en preescolares que han sido víctimas de } \\
\text { agresión sexual, a través de una batería psicodiagnóstica de pruebas proyectivas narrativas y } \\
\text { gráficas (Tesis de Magíster). }\end{array}$ \\
\hline Núñez, L. & 2010 & $\begin{array}{l}\text { Evaluación de daño psíquico en niños preescolares que han sido víctimas de agresión sexual, a } \\
\text { partir del Test de apercepción infantil CAT-A (Tesis de Magíster). }\end{array}$ \\
\hline Sepúlveda, C. & 2010 & $\begin{array}{l}\text { Estudio descriptivo comparativo de las características gráficas en los test de Casa- Árbol- } \\
\text { Persona (HTP) y Persona Bajo la Lluvia de niños preescolares víctimas de agresión sexual. } \\
\text { (Tesis de Magíster). }\end{array}$ \\
\hline
\end{tabular}

